\title{
Protective effect of hydrogen-rich medium against high glucose-induced apoptosis of Schwann cells in vitro
}

\author{
YANG YU ${ }^{1,2}$, XIAOYE MA ${ }^{1,2}$, TAO YANG ${ }^{1,2}$, BO LI $^{2,3}$, KELIANG XIE $^{1,2}$, \\ DAQUAN LIU ${ }^{4}$, GUOLIN WANG ${ }^{1,2}$ and YONGHAO YU ${ }^{1}$ \\ ${ }^{1}$ Department of Anesthesiology, General Hospital of Tianjin Medical University; ${ }^{2}$ Anesthesiology Reseach Institute of Tianjin, \\ Tianjin 300052; ${ }^{3}$ Department of Anesthesiology, The Second Hospital of Tianjin Medical University, Tianjin 300210; \\ ${ }^{4}$ Institute of Acute Abdominal Disease Research Center of Tianjin Integrated Chinese and Western Medicine,
}

Tianjin 300102, P.R. China

Received September 6, 2014; Accepted May 8, 2015

DOI: $10.3892 / \mathrm{mmr} .2015 .3874$

\begin{abstract}
Diabetic peripheral neuropathy (DPN) is considered to be one of the most prevalent and life threatening microvascular diabetic complications. DPN affects up to $50 \%$ of patients with diabetes mellitus and there are currently no efficacious therapeutic strategies available for its treatment. Previous studies have reported that oxidative stress and poly(ADP-ribose) polymerase-1 (PARP-1) may be unifying factors for hyperglycemic injury. The aim of the present study was to investigate the protective effects of hydrogen-rich medium (HM) on high glucose (HG)-mediated oxidative stress, PARP-1 activation and the apoptosis of Schwann cells (SCs) in vitro. The cells were divided into different groups, and were treated for $48 \mathrm{~h}$. Cell viability and apoptosis were evaluated using Cell Counting kit-8 and annexin V/propidium iodide assays, respectively. The concentrations of 8-hydroxy-2-deoxyguanosine (8-OHdG) and peroxynitrite $\left(\mathrm{ONOO}^{-}\right)$were detected using an enzyme-linked immunosorbent assay. The presence of intracellular oxygen free radicals was confirmed using flow cytometric analysis. Colorimetric assays were performed to determine the activity of caspase-3, and western blotting was performed to detect the protein expression levels of PARP-1, cleaved PARP-1, PAR, apoptosis-inducing factor (AIF), B-cell lymphoma 2 (Bcl-2) and $\mathrm{Bcl}-2$-associated $\mathrm{X}$ protein. $\mathrm{HG}$ was found to induce severe oxidative stress and promote the caspase-dependent and caspase-independent apoptosis of SCs. Treatment with $\mathrm{HM}$ inhibited $\mathrm{HG}$-induced oxidative stress by suppressing hydroxyl and $\mathrm{ONOO}^{-}$production, levels of 8-OHdG, caspase-3 activity and apoptosis in the SCs. Furthermore, treatment with HM downregulated the HG-induced release of PAR,
\end{abstract}

Correspondence to: Professor Yonghao Yu, Department of Anesthesiology, General Hospital of Tianjin Medical University, 154 Anshan Road, Heping, Tianjin 300052, P.R. China

E-mail: yuyonghao@126.com

Key words: diabetic peripheral neuropathy, hydrogen, oxidative stress, poly(ADP-ribose) polymerase-1, apoptosis the activation of PARP-1 and nuclear translocation of AIF, and upregulated the expression of Bcl-2 in the SCs. These results indicated that $\mathrm{HM}$ inhibited the $\mathrm{HG}$-induced-oxidative stress-associated caspase-dependent and caspase-independent apoptotic pathways in SCs. Therefore, HM may have potential as a treatment for DPN.

\section{Introduction}

Diabetic peripheral neuropathy (DPN) affects up to $50 \%$ of patients with diabetes mellitus (1) and is considered to be one of the most prevalent and life threatening microvascular diabetic complications. There are currently no efficacious therapeutic strategies available for the treatment of DPN $(2,3)$. DPN is a complex disorder and its exact pathogenesis remains to be fully elucidated; however, various hypotheses have been suggested $(4,5)$. The four predominant pathways associated with DPN are the advanced glycation end products (AGEs) pathway, polyol pathway, protein kinase $\mathrm{C}$ (PKC) pathway and hexosamine pathway (6). Based on previous evidence, oxidative stress, which is induced by the excessive production of reactive oxygen species (ROS), is a key factor in all the above-mentioned pathways (7). Therefore, inhibition of the production of ROS, or alleviation of its detrimental effects, may offer potential for the treatment of DPN.

Molecular hydrogen $\left(\mathrm{H}_{2}\right)$ is the smallest natural gas molecule (8) and it has been suggested that $\mathrm{H}_{2}$ may be promising for extensive used in medical applications, without side effects (9), as a potential oxidation inhibitor. Compared with traditional antioxidants such as vitamin $\mathrm{E}, \mathrm{H}_{2}$ possesses two unique properties. Firstly, it has the ability to readily penetrate cell membranes and rapidly translocate to the nucleus and mitochondria (10). Secondly, it is able to selectively neutralize the more cytotoxic hydroxyl $\left(\mathrm{OH}^{-}\right)$and peroxynitrite $\left(\mathrm{ONOO}^{-}\right)$, without reacting with less potent ROS (11). It is widely accepted that $\mathrm{H}_{2}$ inhalation or the administration of hydrogen-rich saline (HS) may protect against numerous diseases, including type 2 diabetes (12), neurodegenerative disease (13), multiple organ dysfunction syndrome (14), sepsis (15) and atherosclerosis (16). However, whether $\mathrm{H}_{2}$ benefits patients with DPN remains to be elucidated. 
There is increasing evidence suggesting that ongoing hyperglycemia, which invokes oxidative stress events and impairs neurons and peripheral nerve Schwann cells (SCs) (17), is a risk factor in the progression of DPN (18). Excessive ROS-induced DNA breakage can rapidly activate poly(ADP-ribose) polymerase-1 (PARP-1), an enzyme associated with DNA repair, resulting in energy depletion and cell apoptosis $(3,7)$. Notably, overactivation of PARP-1 can cause the substantial release of PAR fragments from the nucleus into the cytoplasm, triggering the caspase-independent apoptotic pathway via translocation of apoptosis-inducing factor (AIF) from the mitochondria to the nucleus (19). However, excessive expression of PARP-1 can also active caspase-3, initiating the caspase-dependent apoptotic pathway (20). PARP-1 itself is degraded by caspase-3 from $116 \mathrm{kDa}$, into two cleavage products ( $89 \mathrm{kDa}$ and $24 \mathrm{kDa})$, thus partially losing its activity (21).

In the present study, SCs were treated with high glucose (HG), in order to produce a cellular research model of DPN. The aim of the present study was to clarify the role of $\mathrm{HG}$ in the process of SC apoptosis, and to examine whether treatment with hydrogen-rich medium (HM) alleviates HG-induced injury in vitro.

\section{Materials and methods}

Preparation of HM. HM was prepared, according to a method previously described by Ohsawa et al (11), with minor modifications. Briefly, $\mathrm{H}_{2}(1 \mathrm{l} / \mathrm{min})$ mixed with air $(1 \mathrm{l} / \mathrm{min})$ was dissolved in low glucose Dulbecco's modified Eagle's medium (DMEM; Gibco Life Technologies, Carlsbad, CA, USA) for $4 \mathrm{~h}$ to reach supersaturation $(\sim 0.6 \mathrm{mM})$, under $0.4 \mathrm{MPa}$ pressure. The saturated HM was then stored in a sealed aluminum bag under atmospheric conditions at $4^{\circ} \mathrm{C}$. HM was freshly prepared every week and was sterilized using $\gamma$-radiation (Co-60 $\gamma$ irradiation facility; Tianjin Institute of Technical Physics, Tianjin, China), in order to maintain a continuous concentration.

Cell culture and treatment. Primary rat SCs (cat. no. R1700; ScienCell Research Laboratories, Carlsbad, CA, USA) were cultured in low glucose DMEM (5.6 mM), supplemented with $5 \%$ fetal bovine serum, $1 \%$ SC growth supplement and $1 \%$ penicillin/streptomycin solution (ScienCell Research Laboratories) in a humidified atmosphere containing $5 \% \mathrm{CO}_{2}$ at $37^{\circ} \mathrm{C}$. Once the cells had reached $\sim 80 \%$ confluence, $0.05 \%$ trypsin-EDTA was added. The second and third passages were selected for the subsequent experiments.

The SCs were randomly divided into five groups, according to the methods of Sun et al (22) with minor modifications. The groups were as follows: Control group (SCs treated with $5.6 \mathrm{mM}$ primary DMEM); $\mathrm{H}_{2}$ group (SCs treated with $0.6 \mathrm{mM} \mathrm{HM}$ ); HG group (SCs treated with $44.4 \mathrm{mM}$ glucose $+50 \mathrm{mM}$ glucose in complete medium); $\mathrm{HG}+\mathrm{H}_{2}$ group (SCs treated with $\mathrm{HG}+0.6 \mathrm{mM} \mathrm{HM}$ ). In order to eliminate osmotic interference, a further group of SCs were treated with $44.4 \mathrm{mM}$ mannitol, which has almost the same osmotic pressure as $44.4 \mathrm{mM}$ glucose. This group was termed the high osmotic control group (mannitol). The cells in each groups were treated for $48 \mathrm{~h}$ at $37^{\circ} \mathrm{C}$.
Cell viability assay. Cell viability was detected using a Cell Counting kit-8 assay (CCK-8; Dojindo Molecular Technologies, Inc., Kuamamoto, Japan). Briefly, the cells were seeded at a density of $2 \times 10^{3}$ per well in 96 -well plates in the different treatment conditions for $48 \mathrm{~h}$, with five parallel wells for each treatment group. Subsequently, $10 \mu \mathrm{l}$ CCK-8 reagent was added to each well and incubated at $37^{\circ} \mathrm{C}$ for another $3 \mathrm{~h}$. Cell density was determined by measuring the absorbance at $450 \mathrm{~nm}$ using a microplate reader (VERSAMax; Molecular Devices, LLC, Sunnyvale, CA, USA), with the cell viability expressed as the percentage of cytoprotection, vs. control group set at $100 \%$.

Cellular apoptosis assay. Apoptosis was determined using an Annexin V-Fluorescein Isothiocyanate (FITC)/Propidium Iodide (PI) kit (Nanjing Keygen Biotech. Co., Ltd., Nanjing, China), according to the manufacturer's instructions. After $48 \mathrm{~h}$ treatment, the SCs in each group were washed twice with pre-cooled phosphate-buffered saline (PBS) and were resuspended at $2 \times 10^{6} / \mathrm{ml}$ in binding buffer (Nanjing Keygen Biotech. Co., Ltd.). A total of $5 \mu 1$ annexin V-FITC and $10 \mu 1$ PI was then added to each well, and the cells were incubated in the dark at room temperature for $15 \mathrm{~min}$. Flow cytometry (FACSCalibur; BD Biosciences, San Diego, CA, USA) was performed to analyze the cells immediately following incubation. CellQuest Pro software v.5.2.1 (BD Biosciences) was used to analyze the data.

Caspase-3 activity assay. An EnzChek Caspase-3 Assay kit (Molecular Probes Life Technologies, Carlsbad, CA, USA) with Z-DEVD-AMC substrate was used to measure caspase-3 activity, according to the manufacturer's instructions. Briefly, after $48 \mathrm{~h}$ treatment of the cells $\left(2 \times 10^{5}\right.$ cells/well $)$ in the different treatment conditions, the cells were collected, lysed with $1 \mathrm{X}$ cell lysis buffer (Molecular Probes Life Technologies) and assayed in a standard black 96-well plate. Once the reactions had been performed with $2 \mathrm{X}$ reaction buffer (Molecular Probes Life Technologies), a fluorescence microplate reader (Fluoroskan Ascent; Thermo Fisher Scientific, Inc., Waltham, MA, USA) was used to measure the fluorescence at $360 \mathrm{~nm}$ excitation and $460 \mathrm{~nm}$ emission wavelengths.

DCFH-DA assay. Intracellular $\mathrm{OH}^{-}$levels were detected using a DCFH-DA assay (Beyotime Institute of Biotechnology, Haimen, China). Briefly, following treatment for $48 \mathrm{~h}$, the cells in each group were harvested. The cells were seeded into a 6 -well plate at $2 \times 10^{6} / \mathrm{ml}$ and labeled with DCFH-DA $(10 \mu \mathrm{M})$ in a humidified atmosphere containing $5 \% \mathrm{CO}_{2}$ in the dark for $20 \mathrm{~min}$ at $37^{\circ} \mathrm{C}$. Following incubation, the cells were washed with PBS and the labeled cells were collected. A flow cytometer (BD Biosciences) was used to detect the fluorescence intensity.

Enzyme-linked immunosorbent assay (ELISA). The concentrations of 8-hydroxy-deoxyguanosine $(8-\mathrm{OHdG})$ and $\mathrm{ONOO}^{-}$were measured using a highly sensitive $8-\mathrm{OhdG}$ Check ELISA kit (Japanese Institute for the Control of Ageing, Shizouka, Japan) and ONOO- ELISA kit (Yueyan Bio, Shanghai, China), respectively. The cellular supernatants were obtained following centrifugation at 3,000 x $\mathrm{g}$ for $15 \mathrm{~min}$ at 
$4^{\circ} \mathrm{C}$, and the levels of 8-OHdG and $\mathrm{ONOO}^{-}$in the supernatants were determined, according to the manufacturer's instructions, with minor modifications, such as to the centrifugation speed. The absorbance was read using the VERSAMax microplate reader.

Western blot analysis. Western blotting was used to detect the relative expression levels of the target proteins. Following treatment of the cells in each group, the cells were lysed with radioimmunoprecipitation buffer (Beijing Solarbio Science \& Technology, Co., Ltd., Beijing, China). The lysates $(50 \mu \mathrm{g} /$ lane) were separated by $10 \%$ SDS-PAGE and were transferred onto polyvinylidene fluoride membranes (EMD Millipore, Billerica, MA, USA). The membranes were blocked with $5 \%$ fat-free milk for $2 \mathrm{~h}$ and were then incubated with the following antibodies: Rabbit polyclonal anti-AIF (1:1,000; cat. no. ab1998; Abcam, Cambridge, MA, USA), rabbit polyclonal anti-PAR (1:1,000; cat. no. 4336-BPC-100; Trevigen Inc., Gaithersburg, MD, USA), rabbit polyclonal anti-cleaved PARP-1/PARP-1 (1:100; cat. no. sc-25780; Santa Cruz Biotechnology, Inc., Dallas, TX, USA), rabbit polyclonal anti-B-cell lymphoma 2 (Bcl-2; 1:100; cat. no. sc-492: Santa Cruz Biotechnology, Inc.), rabbit polyclonal anti-Bcl-2-associated X protein (Bax; 1:100; sc-493; Santa Cruz Biotechnology, Inc.) and anti- $\beta$-actin (1:2,000; cat. no. A5060; Sigma-Aldrich, Shanghai, China), at $4^{\circ} \mathrm{C}$ overnight with mildly consistent agitation. The immunoblots were washed three times with Tris-buffered saline containing $0.05 \%$ Tween $(10 \mathrm{~min} / \mathrm{wash})$, followed by incubation with goat anti-rabbit immunoglobulin G (1:5,000; cat. no. 05557; Sigma-Aldrich) for $2 \mathrm{~h}$ at room temperature. The blots were washed again, as mentioned above, and were treated with a prepared chemiluminescent horseradish peroxidase substrate (EMD Millipore). The blots were visualized using Quantity One software, version 4.5.2 (Bio-Rad Laboratories, Inc., Hercules, CA, USA), and the integrated optical density was analyzed using a Gel-Pro analyzer (Media Cybernetics, Inc., Rockville, MD, USA).

Statistical analysis. The data are presented as the mean \pm standard deviation. One-way analysis of variance with least significant difference comparison were used to analyze differences between the groups. SPSS 21.0 software (IBM SPSS, Armonk, NY, USA) was used to perform the statistical analyses. $\mathrm{P}<0.05$ was considered to indicate a statistically significant difference.

\section{Results}

$H M$ increases the viability of SCs exposed to $H G$. In order to investigate the effects of $\mathrm{HM}$ on cellular viability following treatment with $\mathrm{HG}$, the viability of the SCs was examined using a CCK-8 assay (Fig 1). The viability of the SCs exposed to HG was significantly reduced, compared with the control group $(\mathrm{P}<0.05)$, following treatment for $48 \mathrm{~h}$. Furthermore, treatment with HM improved cell viability under HG conditions $(\mathrm{P}<0.05)$. However, s no significant difference were observed between the mannitol and control groups $(\mathrm{P}>0.05)$.

$H M$ prevents apoptosis of SCs under $H G$ conditions. It is well known that HG-induced oxidative stress promotes apop-

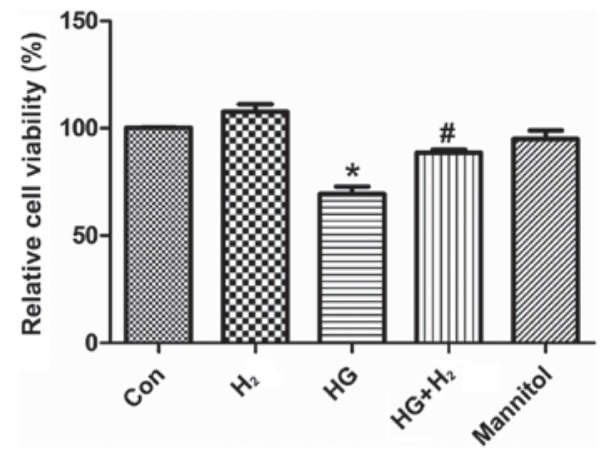

Figure 1. Results of the CCK- 8 assay. The cell viability of the treatment groups following $48 \mathrm{~h}$ treatment was determined using a CCK- 8 assay. The cell viability is expressed as a percentage of each treatment groups, compared with the control group (set at $100 \%$ ). The results are presented as the mean \pm standard deviation ( $\mathrm{n}=5 /$ group). ${ }^{*} \mathrm{P}<0.05$, compared with the Con group; ${ }^{\prime} \mathrm{P}<0.05$, compared with the HG group. CCK-8, Cell Counting kit-8; Con, control; $\mathrm{H}_{2}$, hydrogen; $\mathrm{HG}$, high glucose.

tosis $(18,23)$. To further examine the curative effects of HM on HG-induced apoptosis, the apoptotic rate and caspase-3 activity of the SCs were determined using annexin V-FITC/PI and caspase- 3 activity assays, respectively. The proportion of apoptotic cells in the HG group was markedly increased, compared with the control cells cultured in primary DMEM $(\mathrm{P}<0.05$; Fig. 2A and $\mathrm{B})$. Treatment with HM significantly reduced the percentage of apoptotic cells in the HG condition $(\mathrm{P}<0.05)$. Concordantly, caspase- 3 activity was significantly increased in the HG-treated SCs $(\mathrm{P}<0.05)$, which was alleviated by treatment with $\mathrm{HM}(\mathrm{P}<0.05$; Fig. $2 \mathrm{C})$, whereas treatment with mannitol demonstrated no arresting effects $(\mathrm{P}>0.05)$ on either the apoptotic rate or caspase-3 activity. These results indicated that treatment with HM significantly suppressed HG-induced apoptosis of SCs in vitro.

$\mathrm{HM}$ inhibits the production of $\mathrm{OH}^{-}$and $\mathrm{ONOO}^{-}$under $\mathrm{HG}$ conditions. The levels of intracellular ROS, ONOO ${ }^{-}$and $\mathrm{OH}^{-}$were detected in the treatment groups using ELISA and DCFH-DA assays, respectively. The intracellular levels of $\mathrm{OH}^{-}$ (Fig. 3A and B) and ONOO (Fig. 3C) were higher in the HG group, compared with the control group $(\mathrm{P}<0.05)$. Treatment with HM suppressed the intracellular concentrations of $\mathrm{OH}^{-}$and $\mathrm{ONOO}^{-}$under $\mathrm{HG}$ conditions $(\mathrm{P}<0.05)$, whereas no significant effects of mannitol on levels of ROS were observed $(\mathrm{P}>0.05)$.

HM mitigates HG-induced 8-OHdG levels in SCs. Treatment with HG significantly increased the levels of $8-\mathrm{OHdG}$, a sensitive oxidative stress-induced DNA damage biomarker, compared with the control group (Fig. 4; $\mathrm{P}<0.05$ ). However, treatment with HM markedly reduced the generation of 8-OHdG $(\mathrm{P}<0.05)$, whereas treatment with mannitol had no effect $(\mathrm{P}>0.05)$. These results suggested that HM inhibited oxidative stress-induced DNA damage in the SCs under HG conditions.

Effects of HM on the PARP-l/AIF pathway. To clarify whether HM exhibits a protective effect against HG-induced caspase-independent PARP-1/AIF apoptosis, the acti- 


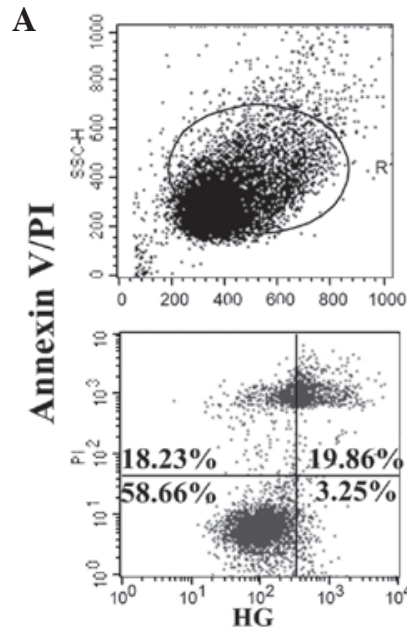

B

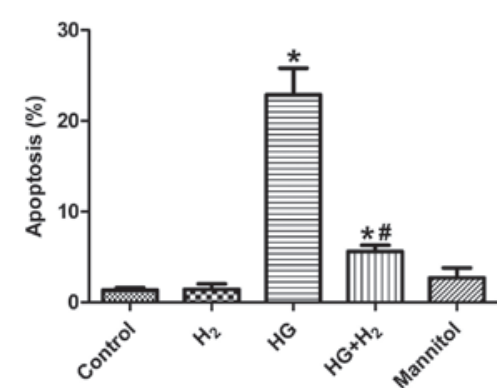

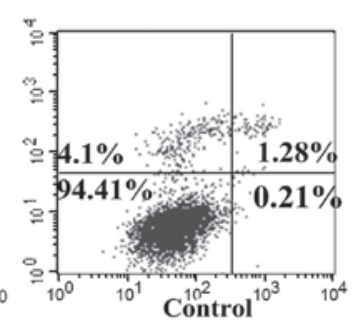

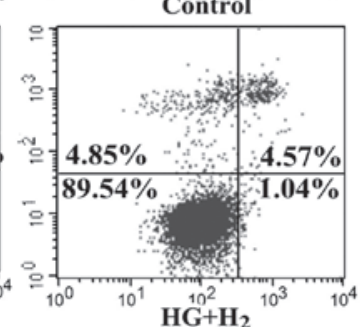

C

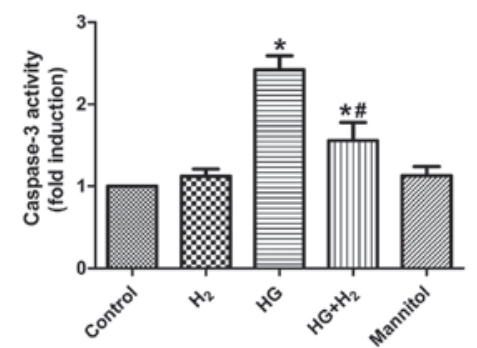

Figure 2. Apoptotic rate of the Schwann cells was determined using annexin V-FITC/PI and caspase-3 activity assays. (A) Representation of the apoptotic cells following flow cytometry, $>5,000$ cells were assessed from each group. The percentage of gated cells (those in the circle) in the lower-right and upper-right quadrants of the plots indicate the proportion of early and late apoptotic cells. Results of the (B) annexin V-FITC/PI and (C) caspase-3 activity assays are expressed as the mean \pm standard deviation ( $\mathrm{n}=3$ /group). ${ }^{*} \mathrm{P}<0.05$, compared with the control group; ${ }^{*} \mathrm{P}<0.05$, compared with the HG group. FITC, fluorescein isothiocyanate; PI, propidium iodide; $\mathrm{H}_{2}$, hydrogen; $\mathrm{HG}$, high glucose.

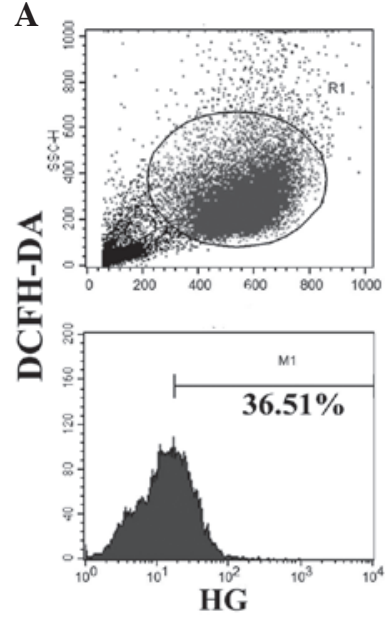

B

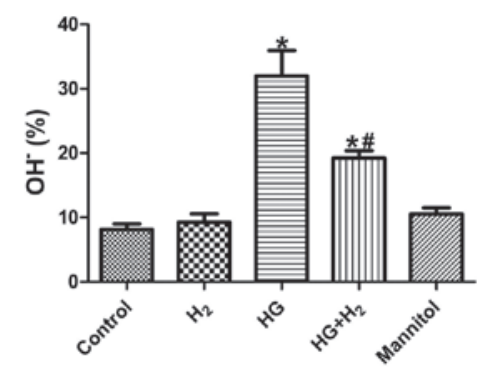

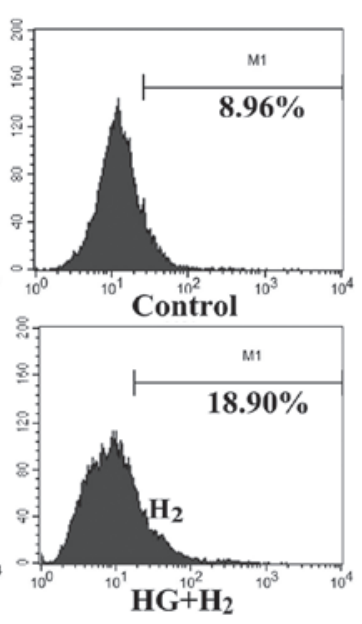

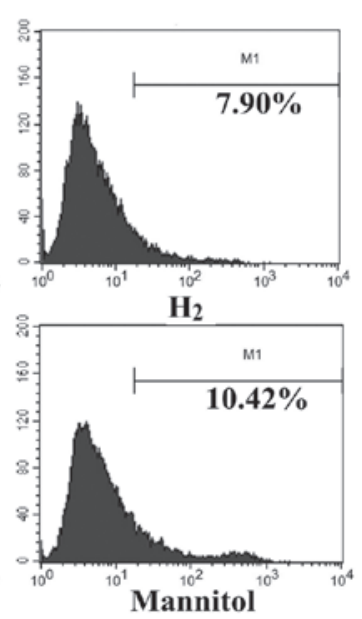

C

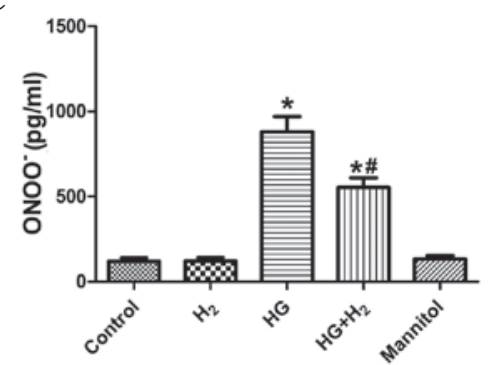

Figure 3. Determination of intracellular $\mathrm{OH}^{-}$and $\mathrm{ONOO}^{-}$levels. Intracellular levels of $\mathrm{OH}^{-}$and $\mathrm{ONOO}^{-}$were detected using flow cytometry and enzyme-linked immunosorbent assays, respectively. (A) Flow cytometric results of the DCFH-DA assay, 20,000 cells were assessed from each group. Quantitative analysis of (B) $\mathrm{OH}^{-}$and (C) $\mathrm{ONOO}^{-}$are expressed as the mean \pm standard deviation ( $\mathrm{n}=3$ /group). ${ }^{*} \mathrm{P}<0.05$, compared with the control group; ${ }^{*} \mathrm{P}<0.05$, compared with the HG group. $\mathrm{H}_{2}$, hydrogen; $\mathrm{HG}$, high glucose; $\mathrm{OH}^{-}$, hydroxyl; $\mathrm{ONOO}^{-}$, peroxynitrite; M1, DCFH-DA labelled cells. 


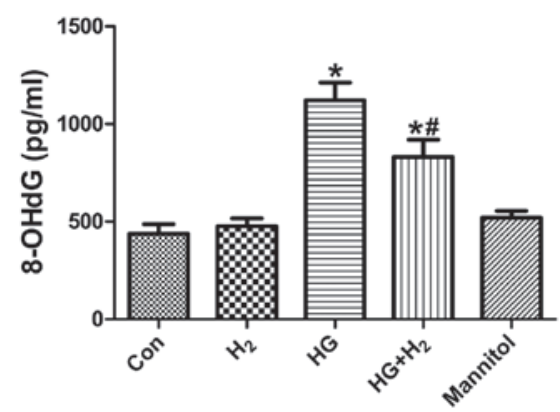

Figure 4. Generation of 8-OHdG. The levels of $8-\mathrm{OHdG}$ were determined in the different treatment groups using an enzyme-linked immunosorbent assay. Data are expressed as the mean \pm standard deviation ( $n=3$ /group). ${ }^{*} \mathrm{P}<0.05$, compared with the control group; ${ }^{*} \mathrm{P}<0.05$, compared with theHG group. 8-OHdG, 8-hydroxy-2-deoxyguanosine; Con, control; $\mathrm{H}_{2}$, hydrogen; $\mathrm{HG}$, high glucose.

vation of PARP-1 (ratio of cleaved-PARP-1/PARP-1), expression of PAR and nuclear translocation of AIF (ratio nuclear AIF/AIF) were detected in each treatment groups using western blot analysis (Fig. 5A). The relative expression levels of cleaved-PARP-1/PARP-1 (Fig. 5B), PAR (Fig. 5C) and nuclear AIF/AIF (Fig. 5D) were markedly increased in the HG group, compared with the control group $(\mathrm{P}<0.05)$. Treatment with HM significantly reduced the activation of PARP-1, expression of PAR and nuclear translocation of AIF $(\mathrm{P}<0.05)$. However, no significant differences in the these proteins were observed between the mannitol and control groups $(\mathrm{P}>0.05)$.

Effects of HM on the expression levels of Bax and Bcl-2 in SCs. The expression levels of pro-apoptotic Bax and anti-apoptotic Bcl-2 were detected using western blot analysis (Fig. 6A) to examine the effects of HM on HG-induced SC apoptosis. After $48 \mathrm{~h}$ treatment, the ratio of $\mathrm{Bax} / \mathrm{Bcl}-2$ was significantly increased in the $\mathrm{HG}$ group, compared with the control group (Fig. 6B; $\mathrm{P}<0.05$ ). However, treatment with HM significantly decreased the HG-induced expression of Bax/Bcl-2 $(\mathrm{P}<0.05)$, and mannitol had no effect $(\mathrm{P}>0.05)$.

\section{Discussion}

It is widely recognized that hyperglycemia, which exists in all patients with diabetes mellitus, is the predominant cause of diabetic complications (6). SCs are a unique type of glial and myelin-forming cell in the peripheral nervous system, which have a critical role in maintaining normal function and morphology and also in the pathogenesis and development of peripheral nerves (24). SCs can rapidly respond to hyperglycemia, generating high levels of antioxidative enzymes, and enhancing antioxidant defense mechanisms to relieve hyperglycemia-induced oxidative stress (25). As injury to the DPN in SCs is reversible (26), it is possible that SCs is a promising target for the treatment of DPN. HG-induced injury has also been widely investigated as a model of chronic disease in vitro (27). Therefore, the present study used SCs cultured under HG conditions as an in vitro cellular model of DPN.

The present study aimed to investigate the protective effects of $\mathrm{H}_{2}$-rich medium on oxidative stress-mediated $\mathrm{SC}$ apoptosis under HG conditions in vitro. The results of the present study indicated that (i) in normal SCs, treatment with $\mathrm{H}_{2}$-rich medium exhibited little effect on cell trauma and viability; (ii) treatment with HG induced severe oxidative stress in SCs with significant injury to the peripheral neural system, and this was significantly improved following treatment with $0.6 \mathrm{mM} \mathrm{HM}$ for $48 \mathrm{~h}$ following HG administration; (iii) HM inhibited oxidative stress-associated DNA damage under hyperglycemic conditions in SCs; and (iv) treatment with HM inhibited HG-induced caspase-dependent and caspase-independent apoptosis in SCs.

Oxidative stress is considered to be an imbalance between the production of ROS and activated anti-oxidative mechanisms in cells (28). Hyperglycemia-induced overproduction of free radicals is considered to be the source of DPN complication through increased glycolysis (29). The production of ROS, including superoxide anions $\left(\mathrm{O}^{-}\right)$and $\mathrm{OH}^{-}$, is unavoidable in all mammalian cells under normal circumstances. In healthy cells, the generation of ROS is closely monitored, whereas during metabolic disturbance, the overproduction of ROS may lead to damage, dysfunction and deletion of nerve fibers in the PNS (30). In DPN, the hyperglycemia-induced overproduction of $\mathrm{O}^{-}$can result in the combination of $\mathrm{NO}$ and $\mathrm{O}^{-}$, resulting in the formation of the $\mathrm{ONOO}^{-}$, a potent antioxidant that can cause cell death (31). In addition to the formation of surplus ROS, hyperglycemia can also induce the dysfunction of organelles and the nucleus, which may lead to the activation of caspase-3, translocation of AIF and cytochrome $c$, and mitochondrial biogenesis and fission (32). Accordingly, the four predominant pathways associated with DPN include the AGE pathway, polyol pathway, PKC pathway and hexosamine pathway (6), all of which lead to programmed cell death. Our previous study demonstrated that, under hyperglycemic conditions, SCs were a target of oxidative damage, and that inhibition of ROS may inhibit the progression of neuropathy (33).

PARP-1 is the most abundant protein in the PARP family, and is a ubiquitous nuclear enzyme, the activation of which in neurons and SCs of the PNS indicates the pathogenesis of diabetic complications (34). Hyperglycemia-associated oxidative stress can initiate excessive DNA single or double strand breaks; however, PARP-1 can respond to this damage by promoting DNA repair procedures via nicotinamide adenine dinucleotide ( $\mathrm{NAD}^{+}$)-dependent poly(ADP-ribos) ylation on histones, or by itself, altering the mitochondrial membrane potential and thus releasing apoptosis-inducing factor from the mitochondria $(35,36)$. Furthermore, excessive PARP-1 activation can result in the generation of increased PAR fragments in the nucleus, which are released into the cytoplasm, promoting AIF nuclear translocation. Therefore, it is possible to directly trigger apoptosis via a caspase-independent pathway (19). Superfluous activation of PARP-1 can markedly consume NAD ${ }^{+}$stores, deplete levels of adenosine triphosphate and destroy the integrity of the oxidative respiratory chain, which can lead to molecular death from necrosis or caspase-dependent apoptosis (37). PARP-1 itself is a substrate of caspase-3, can be decomposed from a $116 \mathrm{kDa}$ protein into two cleavage products ( 89 and $24 \mathrm{kDa}$ ), resulting in partial loss of its activity (21). The present study induced apoptosis of SCs using $50 \mathrm{mM}$ glucose, and demonstrated that HG significantly upregulated the levels of $8-\mathrm{OHdG}$ and increased the expression of cleaved PARP-1, release of PAR, nuclear 
A

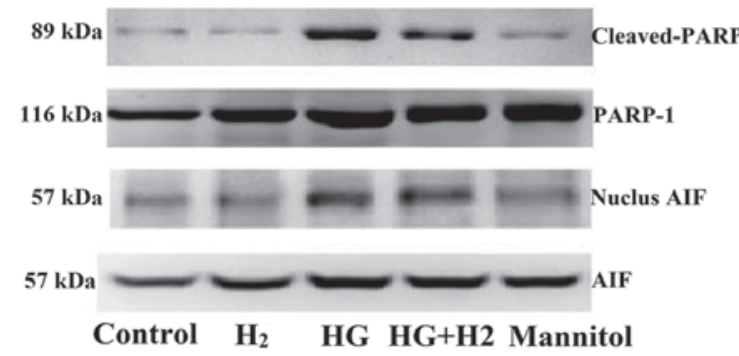

B

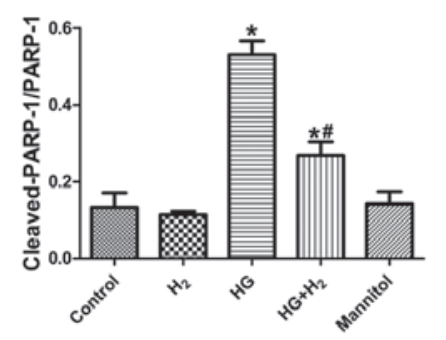

C

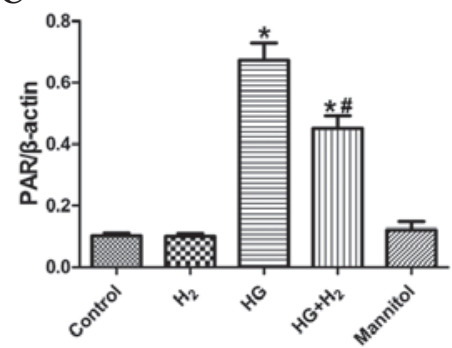

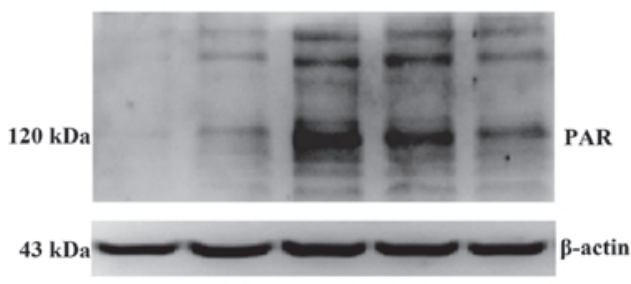

Control $\mathrm{H}_{2} \quad \mathrm{HG} \mathbf{H G}+\mathrm{H}_{2}$ Mannitol

D

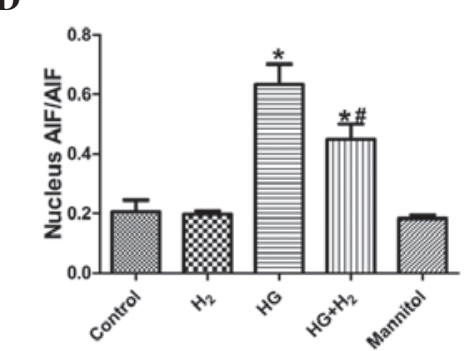

Figure 5. Relative expression levels of the PARP-1/AIF pathway-associated proteins. (A Western blot analysis was used to evaluated the relative expression levels of the PARP-1/AIF pathway-associated proteins. Quantitative analysis of (B) PARP-1 activation, (C) PAR expression and (D) AIF nuclear translocation are all expressed as the mean \pm standard deviation ( $\mathrm{n}=3$ /group). ${ }^{*} \mathrm{P}<0.05$, compared with the control group; ${ }^{*} \mathrm{P}<0.05$, compared with the HG group. PARP-1, poly(ADP-ribose) polymerase-1; AIF, apoptosis-inducing factor; $\mathrm{H}_{2}$, hydrogen; $\mathrm{HG}$, high glucose.

A

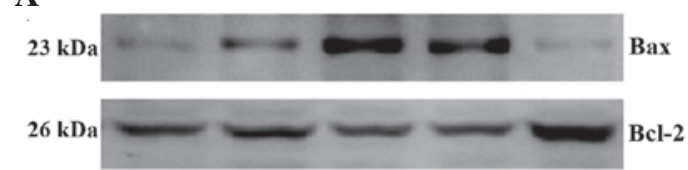

B

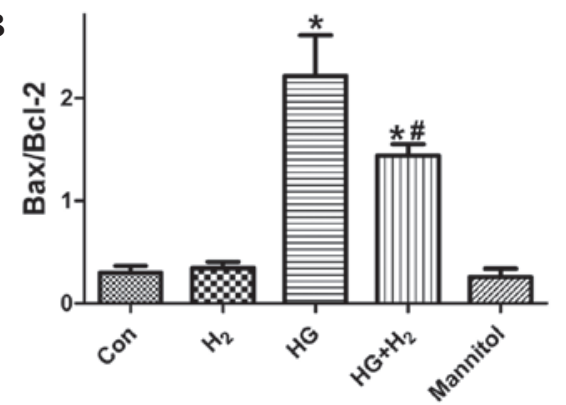

Figure 6. Protein expression levels of Bax and Bcl-2. The relative expression levels of Bax and Bcl-2 were detected in the different treatment groups using western blot analysis. (A) Images of the western blots. (B) An increased $\mathrm{Bax} / \mathrm{Bcl}-2$ ratio was considered to indicate apoptosis of the Schwann cells. Data are expressed as the mean \pm standard deviation ( $\mathrm{n}=3$ /group). ${ }^{*} \mathrm{P}<0.05$, compared with the control group; ${ }^{\prime} \mathrm{P}<0.05$, compared with the HG group. Bcl-2, B-cell lymphoma 2; Bax, Bcl-2-associated X protein; Con, control; $\mathrm{H}_{2}$, hydrogen; HG, high glucose.

tanslocation of AIF and activation of caspase-3. These results indicated that HG induced severe oxidative stress and promoted caspase-independent and caspase-dependent apoptosis of the SCs, both of which are associated with PARP-1.

Since oxidative stress is involved in all DPN pathways, a logical therapeutic strategy may be to prevent oxidative stress by increasing antioxidant defences. Numerous clinical studies have demonstrated that $\mathrm{H}_{2}$ or HS exhibit antioxidative $(9,11,12,38)$, anti-apoptotic (10) and anti-inflammatory $(14,15)$ effects in several disease models. The protective effects of $\mathrm{H}_{2}$ or HS on zymosan-induced organ impairment (14), lipopolysaccharide (LPS)-associated lung injuries (39), LPS-associated sepsis $(15,38,40)$ and ouabain-induced auditory neuropathy $(41)$ have also been observed in vivo. The results of the present study indicated that HM significantly inhibited oxidative damage in the SCs by selectively suppressing the production of $\mathrm{ONOO}^{-}$and $\mathrm{OH}^{-}$, formation of 8-OHdG and activation of PARP-1, leading to a reduction in $\mathrm{SC}$ apoptosis via the caspase-independent and caspase-dependent pathways. In addition, treatment with HM markedly upregulated the expression of anti-apoptotic Bcl-2 and downregulated the expression of pro-apoptotic Bax, indicating that HM protected the SCs from HG-induced apoptosis.

The results of the present study indicated that HG selectively induced the production of ROS and activation of PARP-1, and promoted caspase-dependent and caspase-independent apoptosis of SCs. Furthermore, the findings demonstrated that treatment with HM inhibited HG-induced oxidative stress and the PARP-1 activation-associated apoptosis of SCs by downregulating the caspase-independent and caspase-dependent apoptotic pathways.

\section{Acknowledgements}

This study was supported by the National Natural Science Foundation of China (grant nos. 81372033 to Dr Keliang Xie and 81071533 to Professor Yonghao Yu).

\section{References}

1. Callaghan BC, Cheng HT, Stables CL, et al: Diabetic neuropathy: Clinical manifestations and current treatments. Lancet Neurol 6: 521-534, 2012.

2. Ajroud-Driss S, Christiansen M, Allen JA and Kessler JA: Phase 1/2 open-label dose-escalation study of plasmid DNA expressing two isoforms of hepatocyte growth factor in patients with pain ful diabetic peripheral neuropathy. Mol Ther 21: 1279-1286, 2013.

3. Zenker J, Ziegler D and Chrast R: Novel pathogenic pathways in diabetic neuropathy. Trends Neurosci 36: 439-449, 2013. 
4. Cameron NA, Eaton SE, Cotter MA and Tesfaye S: Vascular factors and metabolic interactions in the pathogenesis of diabetic neuropathy. Diabetologia 44: 1973-1988, 2001.

5. Negi G, Kumar A, Joshi RP, et al: Oxidative stress and diabetic neuropathy: current status of antioxidants. IIOABJ 2: 71-78, 2011

6. Brownlee M: Biochemistry and molecular cell biology of diabetic complications. Nature 414: 813-820, 2001.

7. Obrosova IG: Diabetes and the peripheral nerve. Biochim Biophys Acta 1792: 931-940, 2007.

8. Jiang H, Yu P, Qian DH, et al: Hydrogen-rich medium suppresses the generation of reactive oxygen species, elevates the $\mathrm{Bcl}-2 / \mathrm{Bax}$ ratio and inhibits advanced glycation end product-induced apoptosis. Int J Mol Med 6: 1381-1387, 2013.

9. Terasaki Y, Ohsawa I,Terasaki M, et al: Hydrogen therapy attenuates irradiation-induced lung damage by reducing oxidative stress. Am J Physiol Lung Cell Mol Physiol 301: L415-L426, 2011.

10. Mather P, Salgado KF, Zivin JA and Lapchak PA: A novel approach to screening for new neuroprotective compounds for the treatment of stroke. Brain Res 1173: 117-125, 2007.

11. Ohsawa I, Ishikawa M, Takahashi K, et al: Hydrogen acts as a therapeutic antioxidant by selectively reducing cytotoxic oxygen radicals. Nat Med 13: 688-694, 2007.

12. Fan M, Xu X, Chen L, et al: Protective effects of hydrogen-rich saline against erectile dysfunction in a streptozotocin induced diabetic rat model. J Urol 190: 350-356, 2013.

13. Huang Y, Xie K, Li J, et al: Beneficial effects of hydrogen gas against spinal cord ischemia-reperfusion injury in rabbits. Brain Res 1378: 125-136, 2011.

14. Xie K, Yu Y, Zhang Z, et al: Hydrogen gas improves survival rate and organ damage in zymosan-induced generalized inflammation model. Shock 34: 495-501, 2010.

15. Chen HG, Xie KL, Han HZ, et al: Heme oxygenase-1 mediates the anti-inflammatory effect of molecular hydrogen in LPS-stimulated RAW 264.7 macrophages. Int J Surg 11: 1060-1066, 2013.

16. Ohsawa I, Nishimaki K, Yamagata K, et al: Consumption of hydrogen water prevents atherosclerosis in apolipoprotein $\mathrm{E}$ knockout mice. Biochem Biophys Res Commun 377: 1195-1198, 2008.

17. Viader A, Golden JP, Baloh RH, et al: Schwann cell mitochondrial metabolism supports long-term axonal survival and peripheral nerve function. J Neurosci 31: 10128-10140, 2011.

18. Russell JW, Sullivan KA, Windebank AJ, et al: Neurons undergo apoptosis in animal and cell culture models of diabetes. Neurobiol Dis 6: 347-363, 1999.

19. Yu SW, Wang H, Dawson TM and Dawson VL: Poly(ADP-ribose) polymerase-1 and apoptosis inducing factor in neurotoxicity. Neurobiol Dis 14: 303-317, 2003.

20. Jergens A, Young J, Moore D, et al: Bcl-2/caspase 3 mucosal imbalance favors $\mathrm{T}$ cell resistance to apoptosis in dogs with inflammatory bowel disease. Vet Immunol Immunopathol 158 167-174, 2014

21. Lupachyk S, Shevalye H, Maksimchyk Y, et al: PARP inhibition alleviates diabetes-induced systemic oxidative stress and neural tissue 4-hydroxynonenal adduct accumulation: Correlation with peripheral nerve function. Free Radic Biol Med 50: 1400-1409, 2011.

22. Sun LQ, Chen YY, Wang X, et al: The protective effect of alpha lipoic acid on Schwann cells exposed to constent or intermittent high glucose. Biochem Pharmacol 84: 961-973, 2012.
23. Russell JW, Golovoy D, Vincent AM, Mahendru P, Olzmann JA, Mentzer A and Feldman EL: High glucose-induced oxidative stress and mitochondrial dysfunction in neurons. FASEB J 16 : $1938-1748,2002$

24. Véga C, Martiel JL, Drouhault D, et al: Uptake of locally applied deoxyglucose, glucose and lactate by axons and Schwann cells of rat vagus nerve. J Physiol 546: 551-564, 2003.

25. Askwith T, Zeng W, Eggo MC and Stevens MJ: Oxidative stress and dysregulation of the taurine transporter in high-glucose exposed human Schwann cells: Implications for pathogenesis of diabetic neuropathy. Am J Physiol Endocrinol Metab 297: E620-E628, 2009.

26. Eckeraley L: Role of the Schwann cell in diabetic neuropathy. Int Rev Neurobiol 50: 293-321, 2002.

27. Vincent AM, Russell JW, Low P and Feldman EL: Oxidative stress in the pathogenesis of diabetic neuropathy. Endocr Rev 25: 612-628, 2004

28. Premkumar LS and Pabbidi RM: Diabetic peripheral neuropathy: Role of reactive oxygen and nitrogen species. Cell Biochem Biophys 67: 373-383, 2013.

29. Sun LQ, Xue B, Li XJ, et al: Inhibitory effects of salvianolic acid B on apoptosis of Schwann cells and its mechanism induced by intermittent high glucose. Life Sci 90: 99-108, 2012.

30. Head KA: Peripheral neuropathy: Pathogenic mechanisms and alternative therapies. Altern Med Rev 11: 294-329, 2006.

31. Ame JC, Spenlehauer C and de Murcia G: The PARP superfamily. Bioessays 26: 882-893, 2004.

32. Sun LQ, Zhao J,Zhang TT, et al: Protective effects of Salvianolic acid B on Schwann cells apoptosis induced by high glucose. Neurochem Res 37: 996-1010, 2012.

33. Yu Y, Jiao Y, Li B, et al: Effects of hydrogen on oxidative stress injury induced by high glucose in rat Schwann cells: The relationship with parthanatos. Chin J Anesthesiol 35: 36-39, 2015.

34. Zenker J, Ziegler D and Chrast R: Novel pathogenic pathways in diabetic neuropathy. Trends Neurosci 36: 439-449, 2013.

35. Schreiber V, Dantzer F, Ame JC and de Murcia G: Poly(ADP-ribose): Novel functions for an old molecule. Nat Rev Mol Cell Biol 7: 517-528, 2006.

36. Galluzzi L, Vitale I, Abrams JM, et al: Molecular definitions of cell death subroutines: Recommendations of the Nomenclature Committee on Cell Death 2012. Cell Death Differ 19: 107-120, 2012.

37. Garcia Soriano F, Virág L, Jagtag P, et al: Diabetic endothelial dysfunction: The role of poly(ADP-ribose) polymerase activation. Nat Med 7: 108-113, 2001.

38. Xie K, Yu Y, Pei Y, et al: Protective effects of hydrogen gas on murine polymicrobial sepsis via reducing oxidative stress and HMGB1 release. Shock 34: 90-97, 2010.

39. Xie K, Yu Y, Huang Y, et al: Molecular hydrogen ameliorates lipopolysaccharide-induced acute lung injury in mice through reducing inflammation and apoptosis. Shock 37: 548-555, 2012.

40. Wang X, Li J, Chen Y, et al: p28GANK knockdown-derived reactive oxygen species induces apoptosis through mitochondrial dysfunction mediated by $\mathrm{p} 38$ in HepG2 cells. Int J Oncol 33: 743-750, 2008

41. Qu J, Gan YN, Xie KL, et al: Inhalation of hydrogen gas attenuates ouabain-induced auditory neuropathy. Acta Pharmacol Sin 33: 445-451, 2012 